\title{
Atomic-Scale Investigations of Domain Walls in Polycrystalline $\mathrm{BiFeO}_{3}$
}

\author{
G. Drazic ${ }^{1}$, A. Bencan ${ }^{2}$, D. Damjanovic ${ }^{3}$, and T. Rojac ${ }^{2}$ \\ ${ }^{1 .}$ Department for Materials Chemistry, National Institute of Chemistry, Ljubljana, Slovenia \\ 2. Electronic Ceramics Department, Jozef Stefan Institute, Ljubljana, Slovenia, \\ 3. Group for Ferroelectrics and Functional Oxides, Swiss Federal Institute of Technology - EPFL, \\ Lausanne, Switzerland
}

Multiferroic bismuth ferrite $(\mathrm{BiFeO} 3)$ with Neel and Curie temperatures well above room temperature is a prospective material for high-temperature piezoelectric devices. A serious drawback is its high electrical conductivity, which has recently been discovered to be localized at domain walls (DWs) [1, 2]. While the origin of this local conduction was theoretically explained in terms of accumulation of charged defects at domain walls, no experimental evidence of these defects has been yet provided. Recently, we have reported that in contrast to the usually assumed oxygen vacancies, the dominant defects in $\mathrm{BiFeO}_{3}$ are bismuth vacancies along with compensating electron holes, associated with the presence of $\mathrm{Fe}^{4+}[3]$.

In this contribution we will elaborate two analytical methods (electron-energy loss spectroscopy, EELS, and quantitative high-angle annular dark-field, HAADF, imaging) which lead to the identification of iron (IV) ions and bismuth vacancies accumulated at the domain walls (DW) in $\mathrm{BiFeO}_{3}$. We will further show the results of the domain wall dynamics studied by ex-situ poling experiments. Finally, we will demonstrate that the local domain-wall conductivity can be tailored by material processing.

$\mathrm{BiFeO}_{3}$ (BFO) ceramic samples for atomic-scale analyses were prepared by sintering a mechanochemically activated $\mathrm{Bi}_{2} \mathrm{O}_{3}-\mathrm{Fe}_{2} \mathrm{O}_{3}$ powder mixture at $760^{\circ} \mathrm{C}$ in a packing powder. The samples for the STEM analyses were prepared by grinding, polishing and dimpling and were finally thinned down to electron transparency using Gatan PIPS ion-milling system with simultaneous liquid nitrogen cooling. The structure of domain walls in the BFO ceramics was analysed by atomically resolved Csprobe corrected JEM-ARM 200CF STEM microscope operated at $200 \mathrm{kV}$. Atomic column position were determined using 2D Gaussian fit from HAADF micrographs and normalized Bi-column intensities were measured using a modified approach introduced by LeBeau and Stemmer [4] where average intensity of the background and HAADF STEM detector signal were measured from the HAADF image of detector at non-saturating settings of brightness and contrast.

In a number of regions, a systematic lowering of the intensity of Bi columns at the domain wall was observed. Using quantitative analysis we found at all types of domain walls $\left(71^{\circ}, 109^{\circ}\right.$ and $\left.180^{\circ}\right) \sim 20 \%$ lowering of the column intensity. Figure 1a shows a typical $109^{\circ}$ DW with the corresponding normalized Bi column intensity distribution shown in Figure 1b.

To confirm that the reduction in the intensity (Fig. 1b) is related to Bi concentration we performed EELS analysis on the DW and few nm away from the DW. Background subtracted EELS spectra were integrated in the range from $2580 \mathrm{eV}$ to $2800 \mathrm{eV}$ and plotted as a function of the distance from the DW (Figure 1c.) Relative to the integrated Bi-edge intensity measured away from the DW, a significant signal decrease was clearly observed at the DW, indicating Bi deficiency. This is consistent with the 
assumption that the Bi-column intensity reduction observed in HAADF images is due to the presence of Bi vacancies.

To correlate the Bi column intensities with the amount of Bi vacancies we performed HAADF image simulations with QSTEM code [4] where a multi-slice method and frozen phonon approximation were used. To include the influence of thermal diffuse scattering (TDS), 30 repetitions of calculations per one image were used. Calculations were done on a R3c BFO structural model (ICSD \#97591) in which the concentration of $\mathrm{Bi}$ vacancies was varied in selected columns. From simulated images, using the same approach as for experimental images, we obtained Bi column intensity distribution for different sample thicknesses. By comparing the results obtained from simulated images with those obtained experimentally we estimated a concentration of $\sim 30 \%$ of $\mathrm{Bi}$ vacancies at DWs. These vacancies are likely compensated with the presence of $\mathrm{Fe}^{4+}$ determined from EELS analysis [3].

Experimental details, estimations of errors of the various techniques used during this study, ex-situ poling experiments and the influence of the preparation conditions on the presence and concentration of Bi vacancies and $\mathrm{Fe}^{4+}$ ions will be explained and discussed [6].

\section{References:}

[1] J. Seidel et al, Nature Materials 8 (2009), p. 229.

[2] T. Rojac, et al, Adv. Funct. Mater. 25 (2015), p. 2099.

[3] T. Rojac et al, Nature Materials (2016)

[4] J.M. LeBeau and S. Stemmer, Ultramicroscopy 108 (2008), p.1653.

[5] K. Koch, PhD thesis, Arizona State University, (2002).

[6] This work was supported by the Slovenian Research Agency (J2-7526 and J2-6754).

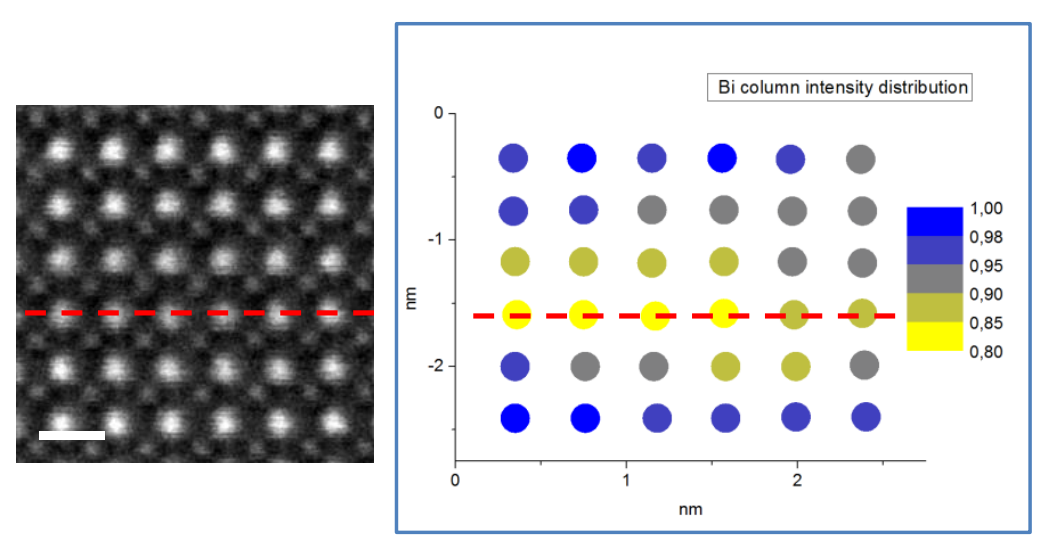

a. b.

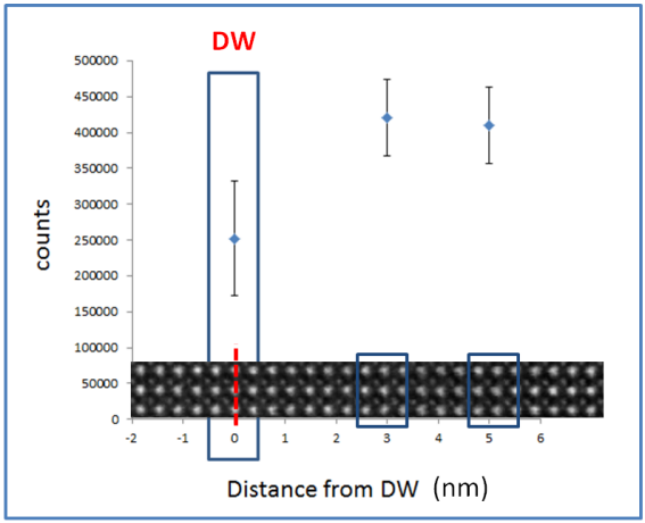

c.

Figure 1. a - HAADF micrograph of a $109^{\circ}$ domain wall region. $\mathrm{b}$ - Bi column intensity distribution measured from image a. $\mathrm{c}$ - integrated intensity of Bi M4,5 edge vs distance from the DW. The inset shows the HAADF image of the area where the spectra were collected. The error bars represents the standard deviations of the measurements. The red dashed line indicates the centre of the DW area. 